\title{
Estimating required information size by quantifying diversity in random-effects model meta-analyses
} Jørn Wetterslev*, Kristian Thorlund, Jesper Brok and Christian Gluud

\author{
Address: Copenhagen Trial Unit, Centre for Clinical Intervention Research, Department 3344, Rigshospitalet, Copenhagen University Hospital, \\ Blegdamsvej 9, DK-2100 Copenhagen Ø, Denmark \\ Email: Jørn Wetterslev* - Wetterslev@ctu.rh.dk; Kristian Thorlund - Kthorlund@ctu.rh.dk; Jesper Brok - jesperb5@hotmail.com; \\ Christian Gluud - Cgluud@ctu.rh.dk \\ * Corresponding author
}

Published: 30 December 2009

BMC Medical Research Methodology 2009, 9:86 doi:10.1 186/147|-2288-9-86

This article is available from: http://www.biomedcentral.com/I47I-2288/9/86

(c) 2009 Wetterslev et al; licensee BioMed Central Ltd.

This is an Open Access article distributed under the terms of the Creative Commons Attribution License (http://creativecommons.org/licenses/by/2.0), which permits unrestricted use, distribution, and reproduction in any medium, provided the original work is properly cited.
Received: 15 May 2009

Accepted: 30 December 2009

\begin{abstract}
Background: There is increasing awareness that meta-analyses require a sufficiently large information size to detect or reject an anticipated intervention effect. The required information size in a meta-analysis may be calculated from an anticipated a priori intervention effect or from an intervention effect suggested by trials with low-risk of bias.
\end{abstract}

Methods: Information size calculations need to consider the total model variance in a metaanalysis to control type I and type II errors. Here, we derive an adjusting factor for the required information size under any random-effects model meta-analysis.

Results: We devise a measure of diversity $\left(D^{2}\right)$ in a meta-analysis, which is the relative variance reduction when the meta-analysis model is changed from a random-effects into a fixed-effect model. $D^{2}$ is the percentage that the between-trial variability constitutes of the sum of the betweentrial variability and a sampling error estimate considering the required information size. $D^{2}$ is different from the intuitively obvious adjusting factor based on the common quantification of heterogeneity, the inconsistency $\left(I^{2}\right)$, which may underestimate the required information size. Thus, $D^{2}$ and $I^{2}$ are compared and interpreted using several simulations and clinical examples. In addition we show mathematically that diversity is equal to or greater than inconsistency, that is $D^{2} \geq R^{2}$, for all meta-analyses.

Conclusion: We conclude that $D^{2}$ seems a better alternative than $R^{2}$ to consider model variation in any random-effects meta-analysis despite the choice of the between trial variance estimator that constitutes the model. Furthermore, $D^{2}$ can readily adjust the required information size in any random-effects model meta-analysis.

\section{Background}

Outcome measures in a single randomised trial or a metaanalysis of several randomised trials are typically dichotomous, especially for important clinical outcomes such as death, acute myocardial infarction, etc. Although metaanalysts cannot directly influence the number of partici- pants in a meta-analysis like trialists conducting a single trial, the assessment of the meta-analytic result depends heavily on the amount of information provided. A limited number of events from a few small trials and the associated random error may be under-recognised sources of spurious findings. If a meta-analysis is conducted before 
reaching a required information size (i.e., the required number of participants in a meta-analysis) it should be evaluated according to the increased risk that the result may represent a chance finding. It has recently been suggested that sample size estimation in a single trial may be less important in the era of systematic review and metaanalysis [1]. Therefore, the reliability of a conclusion drawn from a meta-analysis, despite standardly calculated confidence limits, may depend even more on the number of events and the total number of participants included than hitherto perceived [2-8]. Both numbers determine the amount of available information in a meta-analysis. The information size (IS) required for a reliable and conclusive meta-analysis may be assumed to be at least as large as the sample size (SS) of a single well-powered randomised clinical trial to detect or reject an anticipated intervention effect [2-4].

The estimation of a required information size for a metaanalysis in order to detect or reject an anticipated intervention effect on a binary outcome measure should be considered based on reasonable assumptions. These assumptions may be derived from two kinds of information. Firstly, by anticipating an a priori intervention effect, most appropriately decided at the time when the protocol for a systematic review is prepared. An a priori intervention effect may be estimated by consulting related interventions for the same disease or the same intervention for related diseases suggesting a clinically relevant effect to be detected or ruled out [2-4]. This situation would be almost analogous to the hypothesis testing in a single randomised trial. Secondly, an intervention effect estimated by trials with low-risk of bias in the meta-analysis may represent our best estimate, at a given time point, of a possible intervention effect knowing the available data [5]. This would be a kind of a post hoc analysis of the information needed to detect or reject an intervention effect suggested by data already available. When planning a new trial it may be very important to estimate which IS is needed for the updated meta-analysis to be conclusive. In both instances the estimated required information size may be applied to grade the evidence reported in a cumulative meta-analysis adjusting for the risk of random error due to repetitive testing on accumulating data $[5,6]$. If the number of actually accrued participants falls short of the required IS the meta-analysis may be inconclusive even though the confidence interval is suggestive of a clinical relevant effect or. Because if the confidence interval (or the p-value) is appropriately adjusted with sequential methods, it may no longer show a statistically significant or clinically relevant effect. Conversely, if the actually accrued number of participants supersedes the required information size without the meta-analysis becoming statistically significant we may be able to rule out the anticipated intervention effect size [5].
It is not realistic to assume that the population of the included trials in a meta-analysis is truly homogenous, as it may be in a single clinical trial. Meta-analysis, therefore, should not analyse included participants as if they are coming from one trial [9]. Consequently the difference between obtaining the required $I S$ and $S S$ is rooted in the underlying assumption of between trial variability, and thus, the chosen meta-analytical model.

If the between-trial variability of the outcome measure estimates in a meta-analysis is incorporated into the model using the traditional one-way random-effects model, the required IS will be affected [5]. In this vein, the required $I S$ is a monotonically increasing function of the total variability among the included trials. An estimate of the required IS can therefore be derived once the degree of variability is known or prespecified [5]. The test statistic for heterogeneity in a meta-analysis, the inconsistency factor $\left(I^{2}\right)$ based on Cochran's $Q$ proposed by Higgins and Thompson [10], may seem an obvious quantity to use for this purpose as it allow us to estimate the degree of the variation, which is not covered by assumption of homogeneity [5]. However, $I^{2}$ is derived using a set of general assumptions that may be inappropriate in this context.

In this paper we derive a general expression for the required IS in any random-effects model. We prove the monotone relationship between IS and the degree of total variability in a one-way random-effects meta-analysis. We use our results to define a quantification of diversity $\left(D^{2}\right)$ between included trials in a meta-analysis, which is the relative model variance reduction when the model of pooling is changed from a random-effects model into a fixed-effect model. We analyse and discuss the differences between our definition of diversity, $D^{2}$, and the commonly used measure for heterogeneity, $I^{2}$.

\section{Methods}

\section{I Deriving the required meta-analysis information size and diversity}

If the required IS needed to detect or reject an intervention effect in a meta-analysis should be at least the sample size needed to detect or reject a similar effect in a single trial, then the following scenario applies:

Let $\mu_{\mathrm{F}}$ denote the weighted mean intervention effect to be detected in a fixed-effect model meta-analysis and let $\mu_{R}$ denote the weighted mean intervention effect to be detected in a in a random-effects model meta-analysis using generic inverse variance weighting. The information size $\left(N_{\mathrm{F}}\right)$ needed to reject an intervention effect $\mu_{\mathrm{F}}$ in the fixed-effect model (with a type I error less than $\alpha$, a type II error less than $\beta$, and equal group sizes) becomes $[11,12]$ : 


$$
N_{F}=4 \cdot \frac{\left(Z_{1-\alpha / 2}+Z_{1-\beta}\right)^{2} \cdot v_{F}}{\mu_{F}^{2}}
$$

and the information size $\left(N_{R}\right)$ needed to reject $\mu_{R}$ in the random-effects model (with a type I error less than $\alpha$, a type II error less than $\beta$, and equal group sizes) becomes $[11,12]$ :

$$
N_{R}=4 \cdot \frac{\left(Z_{1-\alpha / 2}+Z_{1-\beta}\right)^{2} \cdot v_{R}}{\mu_{R}^{2}}
$$

Where $v_{F}=\frac{1}{\sum_{1}^{k} w_{i}}$ and $v_{R}=\frac{1}{\sum_{1}^{k} w_{i}^{*}}$ are the variances in the two models with $w_{i}$ and $w_{i}^{*}$ being the weights in the fixedand random-effects model respectively. The ratio of information sizes needed in the two models may be calculated as:

$$
\frac{N_{R}}{N_{F}}=\frac{\mu_{F}^{2}}{\mu_{R}^{2}} \cdot \frac{v_{R}}{v_{F}}
$$

under the assumption that $\mu_{\mathrm{F}}=\mu_{\mathrm{R}}$ it follows that:

$$
\frac{N_{R}}{N_{F}}=\frac{v_{R}}{v_{F}}
$$

or

$$
N_{R}=\frac{v_{R}}{v_{F}} \cdot N_{F}
$$

the relationship between the IS $\left(N_{R}\right)$ for a random-effects model and the $S S\left(N_{F}\right)$ for a fixed-effect model is therefore multiplicative by an adjustment factor $A_{R F}$

$$
I S=A_{R F} \cdot S S
$$

Let $\tau^{2}$ denote the between-trial variance, $k$ the number of trials, and $\sigma_{M}^{2}$ the 'typical' moment-based sampling error within the trials according to Higgins and Thompson [10], then:

$$
A_{R F}=\frac{v_{R}}{v_{F}} \approx \frac{\frac{\tau^{2}+\sigma_{M}^{2}}{k}}{\frac{\sigma_{M}^{2}}{k}}=\frac{\tau^{2}+\sigma_{M}^{2}}{\sigma_{M}^{2}}=\frac{1}{1-I^{2}}
$$

and combining 2.6 and 2.7:

$$
I S=\frac{1}{1-I^{2}} \cdot S S
$$

This yields the intuitive interpretation that the required IS in a random-effects model is a monotone increasing function of the degree of heterogeneity.

\subsection{Limitations of a moment-based 'sampling error' in the definition of heterogeneity, $1^{2}$}

Higgins and Thompson [10] analysed candidate measures of intertrial variability and decided on the inconsistency factor $I^{2}$ under the assumption that all weights $w_{\mathrm{i}}$ were approximately equal, that is, $w_{i}=\frac{1}{\sigma^{2}}$ for all $k$ trials, that is: $\forall i \in\{1, \ldots, k\}$ is $w_{i}=\frac{1}{\sigma^{2}}$. However, this assumption may not be met in many meta-analyses. In the attempt to generalise the use of $I^{2}$ to the situation with trial weights being unequal a 'typical' sampling error $\sigma^{2}$ of the included trials is assumed [10]. To use a 'typical' sampling error $\sigma^{2}$ may not be appropriate in some meta-analyses as weight percentages of the trials easily range from $0.5 \%$ to $50 \%$ or wider without a known distribution (Table 1 and Table 2). The attempt to estimate the trials' 'typical' sampling error as a moment-based sampling error $\sigma_{M}^{2}=\frac{\sum_{1}^{k} w_{i} \cdot(k-1)}{\left(\sum_{1}^{k} w_{i}\right)^{2}-\sum_{1}^{k} w_{i}^{2}}[10]$ may be misleading as it attributes less emphasis to the trials with a very high number of participants and events. $I^{2}$ is interpreted as $I^{2}=\frac{\tau^{2}}{\tau^{2}+\sigma_{M}^{2}}$ and intends to measure the percentage of total meta-analysis variability explained by between-trial variation. In this vein, $I^{2}$ has been interpreted as the between trial variance relative to the sum of the between-trial variance and a 'typical' moment-based sampling error or as it has been phrased: "the between-trial variance rather than the sampling error" [10]. I' achieves some of the desired properties to characterise between-trial variability. However, the concept of a 'typical' sampling error is not relevant if it provides a misleading estimate, seriously distorting the $I^{2}$ calculation or interpretation. If $\sigma_{M}^{2}$ overestimates the sampling error then $I^{2}$ will be underestimated and vice versa. In such instances it may in fact be wise to abandon the concept of a 'typical' sampling error. 
Table I: Meta-analyses examples

\begin{tabular}{|c|c|c|c|c|c|}
\hline Meta-analysis & Title & Intervention & Outcome measure & Number of trials & $\begin{array}{l}\text { Number of } \\
\text { participants }\end{array}$ \\
\hline $\begin{array}{c}\text { Afshari and others } \\
2007[16]\end{array}$ & $\begin{array}{l}\text { Antithrombin III for } \\
\text { critically ill patients }\end{array}$ & Antithrombin III & Mortality & 20 & 3,458 \\
\hline $\begin{array}{l}\text { Al-Inany and others, } \\
2006 \text { [17] }\end{array}$ & $\begin{array}{c}\text { Cycle cancellations due } \\
\text { to poor ovarian } \\
\text { response }\end{array}$ & $\begin{array}{l}\text { Gonadotropin releasing } \\
\text { hormone for assisted } \\
\text { reproductive therapy }\end{array}$ & $\begin{array}{l}\text { Number of cycle } \\
\text { cancellations }\end{array}$ & 13 & 2,543 \\
\hline $\begin{array}{l}\text { Soll and others, } \\
1997[18]\end{array}$ & $\begin{array}{l}\text { Prophylactic surfactant } \\
\text { to prevent morbidity } \\
\text { and mortality in preterm } \\
\text { infants. }\end{array}$ & Surfactant & $\begin{array}{l}\text { Mortality or } \\
\text { pneumothorax }\end{array}$ & 8 & 988 \\
\hline $\begin{array}{l}\text { Wetterslev and Juhl, } \\
2006[19]\end{array}$ & $\begin{array}{c}\text { Effect of perioperative } \beta \text { - } \\
\text { blockade on non-fatal } \\
\text { perioperative AMI }\end{array}$ & $\begin{array}{l}\text { Perioperative } \beta \text { - } \\
\text { blockers for non- } \\
\text { cardiac surgery }\end{array}$ & $\begin{array}{c}\text { Perioperative } \\
\text { myocardial infarction } \\
\text { within } 30 \text { days of } \\
\text { operation }\end{array}$ & 11 & 2,211 \\
\hline $\begin{array}{l}\text { Bury and Tudehope, } \\
2000[20]\end{array}$ & $\begin{array}{l}\text { Effect of antibiotics on } \\
\text { necrotizing enterocolitis } \\
\text { in newborn }\end{array}$ & $\begin{array}{c}\text { Enteral antibiotics in } \\
\text { newborn }\end{array}$ & $\begin{array}{l}\text { Necrotizing } \\
\text { enterocolitis }\end{array}$ & 5 & 458 \\
\hline $\begin{array}{l}\text { Li and others, } \\
2007 \text { [2I] }\end{array}$ & $\begin{array}{l}\text { Intravenous magnesium } \\
\text { for acute myocardial } \\
\text { infarction }\end{array}$ & Magnesium & Mortality & 23 & 72,472 \\
\hline $\begin{array}{l}\text { Meyhoff and others, } \\
2008 \text { [22] }\end{array}$ & $\begin{array}{c}\text { Perioperative ventilation } \\
\text { with } 80 \% \text { versus } 30 \% \\
\text { oxygen during intestinal } \\
\text { surgery }\end{array}$ & $\begin{array}{c}\text { Perioperative } \\
\text { ventilation with } 80 \% \\
\text { oxygen }\end{array}$ & $\begin{array}{l}\text { Wound infection within } \\
15 \text { days of surgery }\end{array}$ & 4 & 989 \\
\hline
\end{tabular}

Table 2: Derived data from meta-analyses examples

\begin{tabular}{|c|c|c|c|c|c|c|c|c|}
\hline $\begin{array}{l}\text { Meta- } \\
\text { analysis }\end{array}$ & $\begin{array}{c}\text { Range of weights } \\
w_{i}(\% \text { weights }) \text { in } \\
\text { the fixed-effect } \\
\text { model }\end{array}$ & $\begin{array}{c}\text { Inconsistency } \\
\left(I^{2}\right) \%\end{array}$ & $\begin{array}{c}\text { Diversity } \\
\left(D^{2}\right) \%\end{array}$ & $\left(D^{2}-I^{2}\right) \%$ & $\begin{array}{l}\text { A priori } \\
\text { relative risk } \\
\text { reduction \% } \\
\quad(R R R)\end{array}$ & $\begin{array}{l}\text { Unadjusted } \\
\text { information } \\
\text { size } \\
\text { (SS) }\end{array}$ & $\begin{array}{l}\text { Heterogeneity- } \\
\text { adjusted } \\
\text { information size } \\
(\text { HIS })\end{array}$ & $\begin{array}{l}\text { Diversity- } \\
\text { adjusted } \\
\text { information } \\
\text { size } \\
\text { (DIS) }\end{array}$ \\
\hline $\begin{array}{l}\text { Afshari and } \\
\text { others } \\
2007[16]\end{array}$ & $\begin{array}{c}0.2-281 \\
(0.04-80 \%)\end{array}$ & 0.0 & 0.0 & 0.0 & 10 & 3,317 & 3,317 & 3,317 \\
\hline $\begin{array}{l}\text { Al-Inany and } \\
\text { others, } \\
2006 \text { [17] }\end{array}$ & $\begin{array}{l}0.2-3.9 \\
(1-18 \%)\end{array}$ & 7.2 & 13.9 & 6.7 & 25 & 3,516 & 3,789 & 4,083 \\
\hline $\begin{array}{c}\text { Soll and others, } \\
1997 \text { [18] }\end{array}$ & $\begin{array}{c}3.6-22.2 \\
(6.2-38.1 \%)\end{array}$ & 22.9 & 37.3 & 14.3 & 60 & 193 & 250 & 307 \\
\hline $\begin{array}{l}\text { Wetterslev } \\
\text { and Juhl, } \\
2006 \text { [19] }\end{array}$ & $\begin{array}{l}0.3-10.4 \\
(1-42 \%)\end{array}$ & 13.4 & 40.5 & 27.1 & 20 & 8,421 & 9,726 & 14,164 \\
\hline $\begin{array}{l}\text { Bury and } \\
\text { Tudehope, } \\
2000 \text { [20] }\end{array}$ & $\begin{array}{l}1.5-9.6 \\
(7-38 \%)\end{array}$ & 40.2 & 57.7 & 17.5 & 45 & 440 & 736 & 1,039 \\
\hline $\begin{array}{c}\text { Li and others, } \\
2007 \text { [2I] }\end{array}$ & $\begin{array}{l}0.24-565.1 \\
(0.02-42 \%)\end{array}$ & 61.9 & 89.9 & 28.0 & 10 & 31,094 & 81,466 & 306,276 \\
\hline $\begin{array}{l}\text { Meyhoff and } \\
\text { others, } \\
2008 \text { [22] }\end{array}$ & $\begin{array}{l}1.3-15.2 \\
(4-47 \%)\end{array}$ & 74.2 & 79.4 & 5.2 & 30 & 1,699 & 6,581 & 8,239 \\
\hline
\end{tabular}


If the focus is shifted towards a sufficient IS estimation, then adjusting factors based on $I^{2}$ calculated from a moment-based sampling error may be insufficient. We therefore suggest to consider an alternative adjusting factor to obtain an adequate estimation of the required IS.

\subsection{Defining and implementing a measure of diversity} Assume we are interested in showing or rejecting a significant intervention effect, $\mu$, regardless of the choice of meta-analysis model (fixed or random). That is, assume $\mu$ $=\mu_{\mathrm{F}}=\mu_{\mathrm{R}}$. We then define diversity $\left(D^{2}\right)$ as the quantity compelled to satisfy the following equation:

$$
A_{R F}=\frac{v_{R}}{v_{F}}=\frac{\sum_{1}^{k} w_{i}}{\sum_{1}^{k} w_{i}^{*}}=\frac{1}{1-D^{2}}
$$

Solving the equation with respect to $D^{2}$ we get the definition of $D^{2}$ explicitly:

$$
D^{2}=1-\frac{v_{F}}{v_{R}}=\frac{v_{R}-v_{F}}{v_{R}}=1-\frac{\sum_{1}^{k} w_{i}^{*}}{\sum_{1}^{k} w_{i}}=1-\frac{\sum_{1}^{k}\left(w_{i}^{-1}+\tau^{2}\right)^{-1}}{\sum_{1}^{k} w_{i}} .
$$

As long as we do not know what the difference between $I^{2}$ and $D^{2}$ covers, knowing now from 2.9 that $D^{2}$ reflects the total relative variance expansion changing from a fixedeffect into a random-effects model meta-analysis, we find it wise to denote $D^{2}$ diversity instead of just another calculation of heterogeneity. $A_{R F}$ will be an adjustment of $N_{F}$ to $N_{R}$ taking into account the total variance expansion changing from a fixed-effect into a random-effects model. Hereby, $D^{2}$ expresses the relative variance reduction when the model of meta-analysis is changed from a randomeffects model into a fixed-effect model. $D^{2}$ is the percentage of change in variance when the model is changed. $D^{2}$ becomes exactly the proportion that the between-trial variance component $\left(\tau^{2}=k \cdot\left(V_{R}-V_{F}\right)\right)$ constitutes of the sum of variances $\left(\tau^{2}+\sigma_{D}^{2}=v_{R} \cdot k\right)$ in the variance component model if and only if $\sigma_{D}^{2}$ (a sampling error originating from diversity or the required information size) is defined as:

$$
\sigma_{D}^{2}=\frac{\tau^{2} \cdot v_{F}}{v_{R}-v_{F}} .
$$

Diversity can then be expressed as:

$$
D^{2}=\frac{v_{R}-v_{F}}{v_{R}}=\frac{\tau^{2}}{\tau^{2}+\sigma_{D}^{2}}
$$

This way, $D^{2}$ in a meta-analysis may become a central measure of the between-trial variability relative to the sum of the between-trial variability with an estimate of the sampling error basically originating from the required information size.

As such, $D^{2}$ is able to quantify the relative model variance change from a random-effects into a fixed-effect model. More importantly $D^{2}$, in contrast to $I^{2}$, is not based on underlying assumptions of a 'typical' sampling error that are violated in most meta-analyses. $D^{2}$ is the percentage of the total variance (the sum of between trial variance and sampling error), in a random-effects model, contributed by the between trial variance.

\subsection{Simulating meta-analyses}

In our simulations, we considered meta-analyses with $k=$ 6 and $k=20$ trials. For each $k$, we considered the four combinations from two different average control event proportions, $(P C)$ of $10 \%$ and $30 \%$, and two true values of the overall effect in terms of odds ratios of 1 and 0.7. The above values were selected aiming to cover different plausible meta-analytic scenarios. In total, these values make up for 8 simulation scenarios.

For each combination of the above mentioned variables we generated data for $k 2 \times 2$ tables. For all $k$ trials, within group sample sizes were determined by sampling an integer between 20 and 500 participants. Group sizes were equal in each simulated trial. We drew the trial specific control group event rate, $P C_{i}$, from a uniform distribution, $P C_{i} \sim \mathrm{U}(P C-0.15, P C+0.15)$. We drew the number of observed events in the control group from a binomial distribution $e_{i C} \sim \operatorname{bin}\left(n_{i}, P C_{i}\right)$. For each meta-analysis scenario we varied the degree of heterogeneity by sampling the between-rial standard deviation, $\tau$ (not the between-trial variance $\left.\tau^{2}\right)$, from a uniform distribution, $\tau \sim U\left(10^{-10}\right.$, $S Q R T(0.60))$. We simulated the underlying true trial intervention effects, as log odds ratio $\ln \left(O R_{i}\right) \sim N\left(O R, \tau^{2}\right)$, where $O R$ is the true intervention effect expressed as an odds ratio. We drew the observed number of events in the intervention group from a binomial distribution $e_{i E^{\sim}} \operatorname{bin}\left(n_{i}, P E_{i}\right)$, where $P E_{i}=P C_{i} \exp \left(\ln \left(O R_{i}\right)\right) /\left(1-P C_{i}+\right.$ $\left.P C_{i} \exp \left(\ln \left(O R_{i}\right)\right)\right)$

For all meta-analysis scenarios we simulated 10,000 metaanalyses and for each of these we calculated the $I^{2}=\frac{Q-(k-1)}{Q}$ and the $D^{2}=\frac{v_{R}-v_{F}}{v_{R}}$. For each scenario we 
plotted $D^{2}$ against $I^{2}$ and incorporated the line of unity in the scatter-plot.

\subsection{Selection of meta-analyses examples}

We selected traditional random-effects meta-analyses to cover a range of inconsistency $I^{2}$ from $0 \%$ to $100 \%$ and to come from a wide range of medical research fields.

\section{Results}

\section{I The relationship between diversity, $D^{2}$, and} heterogeneity, $I^{2}$

We want to show that $D^{2} \geq I^{2}$ for all meta-analyses. This is true if and only if:

$$
D^{2}=\frac{\tau^{2}}{\tau^{2}+\sigma_{D}^{2}} \geq \frac{\tau^{2}}{\tau^{2}+\sigma_{M}^{2}}=I^{2}
$$

According to a special case of the Chebyshev's inequality [13] we arrange the weights so $w_{1} \geq w_{2} \geq \ldots \ldots . w_{k}$, for any $k$ $\geq 0$ we then get that:

$$
\left(w_{1}+\ldots \ldots \ldots+w_{k}\right) \cdot\left(w_{1}+\ldots \ldots \ldots+w_{k}\right) \leq k \cdot\left(w_{1} \cdot w_{1}+\ldots . .+w_{k} \cdot w_{k}\right)
$$

and hence:

$$
\left(\sum_{i=1}^{k} w_{i}\right)^{2} \leq k \cdot \sum_{i=1}^{k} w_{i}^{2}
$$

and therefore

$$
k \cdot\left(\left(\sum_{i=1}^{k} w_{i}\right)^{2}-\sum_{i=1}^{k} w_{i}^{2}\right) \leq(k-1) \cdot\left(\sum_{i=1}^{k} w_{i}\right)^{2}
$$

and subsequently:

$$
\frac{k}{\sum_{i=1}^{k} w_{i}} \leq \frac{(k-1) \cdot \sum_{i=1}^{k} w_{i}}{\left(\sum_{i=1}^{k} w_{i}\right)^{2}-\sum_{i=1}^{k} w_{i}^{2}}
$$

We remember that Takouche et al. [14] proposed an estimate of a 'typical' sampling error $\sigma_{T}^{2}=k \cdot v_{F}$, which yields the following relationship between $\sigma_{T}^{2}$ and $\sigma_{M}^{2}$ :

$$
\sigma_{T}^{2}=\frac{k}{\sum_{i=1}^{k} w_{i}} \leq \frac{(k-1) \cdot \sum_{i=1}^{k} w_{i}}{\left(\sum_{i=1}^{k} w_{i}\right)^{2}-\sum_{i=1}^{k} w_{i}^{2}}=\sigma_{M}^{2}
$$

So it follows from (3.5) that $\sigma_{T}^{2} \leq \sigma_{M}^{2}$ for all meta-analyses. Furthermore if we apply Chebyshev's inequality [13] arranging the weights $w_{1}^{*} \geq w_{2}^{*} \geq \ldots \ldots \geq w_{k}^{*}$ and at the same time $w_{1} \geq w_{2} \geq \ldots \ldots w_{k}$ then:

$\left(w_{1}^{*}+\ldots \ldots \ldots+w_{k}^{*}\right) \cdot\left(w_{1}+\ldots \ldots \ldots+w_{k}\right) \leq k \cdot\left(w_{1}^{*} \cdot w_{1}+\ldots . .+w_{k}^{*} \cdot w_{k}\right)$ and as the random-effects weights are $w_{i}^{*}=\frac{1}{\sigma_{i}^{2}+\tau^{2}}$ and the fixed-effect weights are $w_{i}=\frac{1}{\sigma_{i}^{2}}$ we get:

$\left(\frac{1}{\sigma_{1}^{2}+\tau^{2}}+\ldots+\frac{1}{\sigma_{k}^{2}+\tau^{2}}\right) \cdot\left(\frac{1}{\sigma_{1}^{2}}+\ldots+\frac{1}{\sigma_{k}^{2}}\right) \leq k \cdot\left(\frac{1}{\sigma_{1}^{2}} \cdot \frac{1}{\sigma_{1}^{2}+\tau^{2}}+\ldots+\frac{1}{\sigma_{k}^{2}} \cdot \frac{1}{\sigma_{k}^{2}+\tau^{2}}\right)$

and hence:

$\frac{1}{\left(\frac{1}{\sigma_{1}^{2}+\tau^{2}}+\ldots+\frac{1}{\sigma_{k}^{2}+\tau^{2}}\right) \cdot\left(\frac{1}{\sigma_{1}^{2}}+\ldots+\frac{1}{\sigma_{k}^{2}}\right)} \geq \frac{1}{k \cdot\left(\frac{1}{\sigma_{1}^{2}} \cdot \frac{1}{\sigma_{1}^{2}+\tau^{2}}+\ldots+\frac{1}{\sigma_{k}^{2}} \cdot \frac{1}{\sigma_{k}^{2}+\tau^{2}}\right)}$

which leads to:

$\frac{\tau^{2} \cdot \sum^{k} \frac{1}{\sigma_{i}^{2} \cdot\left(\sigma_{i}^{2}+\tau^{2}\right)}}{\left(\frac{1}{\sigma_{1}^{2}+\tau^{2}}+\ldots+\frac{1}{\sigma_{k}^{2}+\tau^{2}}\right) \cdot\left(\frac{1}{\sigma_{1}^{2}}+\ldots+\frac{1}{\sigma_{k}^{2}}\right)} \geq \frac{\tau^{2} \cdot \sum \frac{1}{\sigma_{i}^{2} \cdot\left(\sigma_{i}^{2}+\tau^{2}\right)}}{k \cdot\left(\frac{1}{\sigma_{1}^{2}} \cdot \frac{1}{\sigma_{1}^{2}+\tau^{2}}+\ldots+\frac{1}{\sigma_{k}^{2}} \cdot \frac{1}{\sigma_{k}^{2}+\tau^{2}}\right)}$

and subsequently:

$k \cdot\left(\frac{\tau^{2} \cdot \frac{1}{1} \frac{1}{\sigma_{i}^{2} \cdot\left(\sigma_{i}^{2}+\tau^{2}\right)}}{\left(\frac{1}{\sigma_{1}^{2}+\tau^{2}}+\ldots+\frac{1}{\sigma_{k}^{2}+\tau^{2}}\right) \cdot\left(\frac{1}{\sigma_{1}^{2}}+\ldots+\frac{1}{\sigma_{k}^{2}}\right)} \geq k \cdot \frac{\tau^{2} \cdot\left(\frac{1}{\sigma_{1}^{2}} \cdot \frac{1}{\sigma_{1}^{2}+\tau^{2}}+\ldots+\frac{1}{\sigma_{k}^{2}} \cdot \frac{1}{\sigma_{k}^{2}+\tau^{2}}\right)}{k \cdot\left(\frac{1}{\sigma_{1}^{2}} \cdot \frac{1}{\sigma_{1}^{2}+\tau^{2}}+\ldots+\frac{1}{\sigma_{k}^{2}} \cdot \frac{1}{\sigma_{k}^{2}+\tau^{2}}\right)}=\tau^{2}\right.$

and since:

$k \cdot \frac{\tau^{2} \cdot \sum_{1}^{k} \frac{1}{\sigma_{i}^{2} \cdot\left(\sigma_{i}^{2}+\tau^{2}\right)}}{\left(\frac{1}{\sigma_{1}^{2}+\tau^{2}}+\ldots+\frac{1}{\sigma_{k}^{2}+\tau^{2}}\right) \cdot\left(\frac{1}{\sigma_{1}^{2}}+\ldots+\frac{1}{\sigma_{k}^{2}}\right)}=k \cdot\left(\frac{1}{\sum_{1}^{k} w_{i}^{*}}-\frac{1}{\sum_{1}^{k} w_{i}}\right)$

it follows that:

$$
k \cdot\left(\frac{1}{\sum_{1}^{k} w_{i}^{*}}-\frac{1}{\sum_{1}^{k} w_{i}}\right)=k \cdot\left(v_{R}-v_{F}\right) \geq \tau^{2}
$$

and therefore: 
Remembering the definitions of $\sigma_{D}^{2}$ and $\sigma_{T}^{2}$ lead to:

$$
\sigma_{D}^{2}=\frac{\tau^{2} \cdot v_{F}}{v_{R}-v_{F}} \leq k \cdot v_{F}=\sigma_{T}^{2}
$$

and it appears from (3.7) that $\sigma_{D}^{2} \leq \sigma_{T}^{2}$ for all meta-analyses. As we have already shown in (3.5) that $\sigma_{T}^{2} \leq \sigma_{M}^{2}$ it becomes clear that $\sigma_{D}^{2} \leq \sigma_{M}^{2}$ in all meta-analyses. As $\sigma_{D}^{2} \leq \sigma_{T}^{2} \leq \sigma_{M}^{2}$ and with $T^{2}=\frac{\tau^{2}}{\tau^{2}+\sigma_{T}^{2}}$ it follows that:

$$
D^{2}=\frac{\tau^{2}}{\tau^{2}+\sigma_{D}^{2}} \geq \frac{\tau^{2}}{\tau^{2}+\sigma_{T}^{2}} \geq \frac{\tau^{2}}{\tau^{2}+\sigma_{M}^{2}}=I^{2}
$$

and, finally, $D^{2} \geq T^{2} \geq I^{2}$ in all meta-analyses.

\subsection{Some useful properties of $D^{2}$}

Higgins and Thompson [10] specified three criteria that should be met by any quantification of variability between trials included in a meta-analysis: 1) the quantity should be a monotonically increasing function of the between-study variance, $\tau^{2} ; 2$ ) the quantity should be scale invariant; and 3) the quantity should be independent of the number of included trials. It is easily verified that the $D^{2}$ fulfils the first two of these criteria. The third criterion may not be fulfilled, even by $I^{2}$ (simulations by $\mathrm{K}$ Thorlund, personal communication). However, $D^{2}$ becomes independent of the numbers of trials included in the meta-analysis, to the same degree as $\frac{v_{R}}{v_{F}}$, because $D^{2}$ is a transformation of $\frac{v_{R}}{v_{F}}$ fulfilling the criterion according to Higgins and Thompson [10]. Furthermore, it is easy to show that:

$$
\frac{v_{R}-v_{F}}{v_{F}}=\frac{D^{2}}{1-D^{2}}
$$

demonstrating that the percentage of increase in variance when the model of meta-analysis is changed from a fixedeffect model into a random-effects model can, of course, also be expressed in terms of diversity.

It is equally clear that $D^{2}$ is always $\geq 0$ as well as being $<1$. $D^{2}$ is a fraction between 0 and $100 \%$ because:

$$
\sum_{1}^{k} w_{i}^{*}=\sum_{1}^{k}\left(w_{i}^{-1}+\tau^{2}\right)^{-1}=\sum_{1}^{k} \frac{w_{i}}{1+w_{i} \cdot \tau^{2}} \leq \sum_{1}^{k} w_{i}
$$

as $\left(1+w_{\mathrm{i}} \cdot \tau^{2}\right) \geq 1$ for all $i$ and for all estimators of $\tau^{2}$ including the DerSimonian-Laird estimator $\hat{\tau}^{2}=\max \left\{0, \frac{(Q-k+1) \cdot \sum_{1}^{k} w_{i}}{\left(\sum_{1}^{k} w_{i}\right)^{2}-\sum_{1}^{k} w_{i}^{2}}\right\}[15]$ with $\hat{\tau}^{2}$ being at least greater than or equal to 0 .

Furthermore, $D^{2}=I^{2}$ when and only when all the weights $w_{\mathrm{i}}$ in the fixed-effect model are equal. $D^{2}$ is approximately equal to $I^{2}$ if:

$$
\sigma_{D}^{2} \approx \sigma_{M}^{2}=\frac{(k-1) \cdot \sum^{k} w_{i}}{\left(\sum_{1}^{k} w_{i}\right)^{2}-\sum_{1}^{k} w_{i}^{2}}
$$

Furthermore, $D^{2}=0$ when and only when $I^{2}=0$ because $I^{2}$ $=0$ when and only when $\tau^{2}=0$ the latter making $\frac{v_{F}}{v_{R}}=1$ and hence $D^{2}=1-\frac{v_{F}}{v_{R}}=0$.

$$
D^{2}=1-\frac{v_{F}}{v_{R}}=1-\frac{\sum_{1}^{k} w_{i}^{*}}{\sum_{1}^{k} w_{i}}=\frac{\sum_{1}^{k} w_{i}-\sum_{1}^{k} w_{i}^{*}}{\sum_{1}^{k} w_{i}}
$$

\subsection{Simulations of meta-analyses}

We performed 8 simulation scenarios showing that $D^{2}$ always exceeds $I^{2}$ despite any assumptions. Meta-analyses with all weights being equal corresponding to $D^{2}=I^{2}$ were rare. The pattern of data showed a greater degree of scatter in the scenarios where $k=6$. The results of the simulations of 10000 meta-analyses according to the combinations of $O R=0.70, O R=1.00$ and $P C=30 \%$ with 6 and 20 trials, respectively, are presented in figure 1 and figure 2 . As seen $D^{2}$ exceeds $I^{2}$ for all the simulated meta-analyses independent of the chosen $O R$ and number of trials in the meta-analyses.

\subsection{Examples}

We used the expression of $D^{2}$ to calculate this quantity in seven traditional random-effects meta-analyses [16-22] listed in Table 1 . These meta-analyses cover a range of inconsistency, $I^{2}$, from $0 \%$ to $74.2 \%$ and come from different medical research fields: intensive care [16], assisted reproductive technology [17], perioperative medicine $[19,22]$, neonatology $[18,20]$, and cardiology [21]. The results of the calculations of $I^{2}, D^{2}$, inconsistency-adjusted information size HIS $\left(H I S=\frac{S S}{1-I^{2}}\right)$, and diversity- 


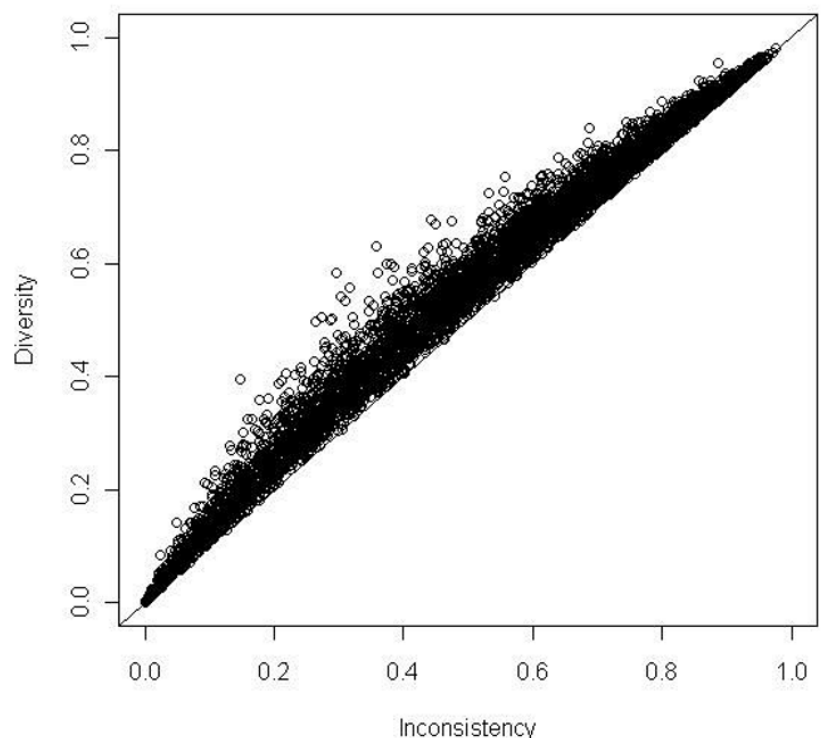

Figure I

Diversity $\left(D^{2}\right)$ compared to inconsistency $\left(I^{2}\right)$ in 10,000 simulations of meta-analyses with number of trials included $\boldsymbol{k}=\mathbf{6}$. Odds ratio $=1.00$ and proportion of events in control group $P C=0.30$. Meta-analyses depicted as open circles. $D^{2}$ nears asymptotically to $I^{2}$ when heterogeneity nears $0 \%$ or $100 \%$. Line of unity, $D^{2}=p^{2}$ black line.

adjusted information size DIS ( DIS $\left.=\frac{S S}{1-D^{2}}\right)$ from these meta-analyses are shown in Table 2 . The range of the calculated unadjusted SS range from 440 to 31,094 participants.

Figure 3 shows the relationship between $D^{2}, I^{2}$, and unity. All the meta-analyses examples are shown as open circles above the line of unity as $D^{2} \geq I^{2}$. The difference $\left(D^{2}-I^{2}\right)$ increases with heterogeneity until a certain point, after which the difference again regresses to 0 .

\section{Discussion}

Using a mathematical derivation, meta-analyses simulations, and examples of meta-analyses we derive a concept of diversity, $D^{2}$. $D^{2}$ may be used for adjustment of the required information size in any random-effects model meta-analysis once the between trial variance is estimated. Focusing on the required information size estimation in a random-effects meta-analysis, $D^{2}$ seems less biased compared to $I^{2}$. The $D^{2}$ is directly constructed to fulfil the requirements of the information size calculation and is subsequently independent of any 'typical' a priori sampling error estimate, whereas the $I^{2}$ is influenced by an $a$ priori 'typical' sampling error estimate. We therefore find that it is possible and appropriate taking $D^{2}$ into consideration to calculate the required IS in meta-analyses as DIS.

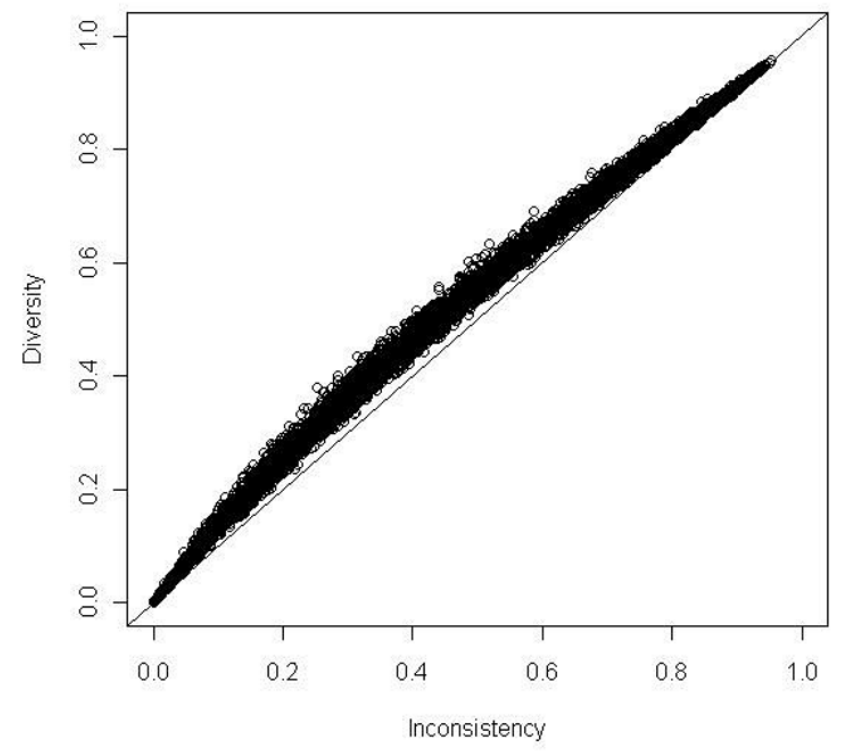

Figure 2

Diversity $\left(D^{2}\right)$ compared to inconsistency $\left(I^{2}\right)$ in I 0,000 simulations of meta-analyses with number of trials included $\boldsymbol{k}=\mathbf{2 0}$. Odds ratio $=0.70$ and proportion of events in control group $P C=0.30$. Meta-analyses depicted as open circles. $D^{2}$ nears asymptotically to $I^{2}$ when heterogeneity nears $0 \%$ or $100 \%$. Line of unity, $D^{2}=1^{2}$ black line.

DIS has several advantages. It measures the required IS needed to preserve the anticipated risk of type I and type II errors in a random-effects model meta-analysis. DIS considers total variance change when the model shifts from a fixed-effect into a random-effects model. DIS is a model dependent and derived estimate of the required IS. The adjustment is dependent only on the anticipated intervention effect and on the model used to incorporate the between-trial variance estimate $\hat{\tau}^{2} . D^{2}$ applies to random-effects models other than that proposed by DerSimonian-Laird [16] as long as the between-trial estimator, $\hat{\tau}^{2}$, is specified. The adjustment of IS does not depend on the level of type I and II errors, as $\left(Z_{1-\alpha / 2}+Z_{1-\beta}\right)^{2}$ is levelled out during the derivation of the adjustment factor $A_{R F}$ (see equation 2.1, 2.2, and 2.5). The relationship $D^{2} \geq I^{2}$ in all the simulations and in all the examples (shown as points above the line of unity in figure 1, 2, and 3) are in accordance with the properties of $D^{2}$ compared to $I^{2}$ derived in section 3.1 .

There are limitations of DIS. Like HIS the use of DIS cannot compensate for systematic bias such as selection bias, allocation bias, reporting bias, collateral intervention bias, and time lag bias [5,23-28]. Furthermore, DIS is always greater than or equal to HIS, which may emphasise 


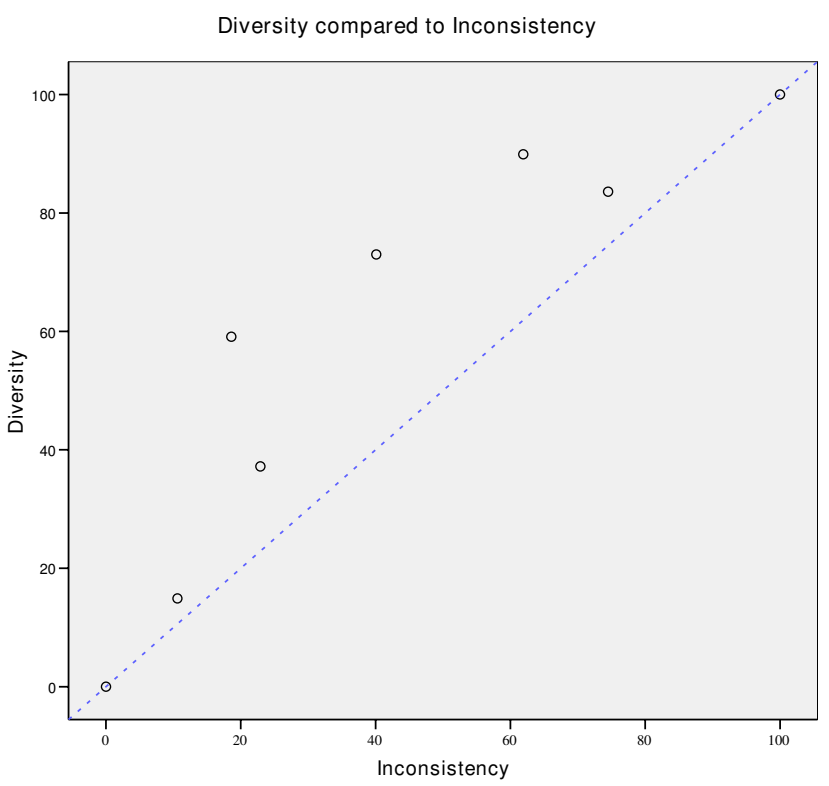

Figure 3

Diversity $\left(D^{2}\right)$ in \% compared to inconsistency $\left(I^{2}\right)$ in $\%$ in seven meta-analyses (see Table I) depicted as open circles. The open circles indicate that $D^{2}$ is always equal to or larger than $R^{2}$. $100 \%$ heterogeneity is impossible and the upper right point is just to illustrate that $D^{2}$ nears asymptotically to $I^{2}$ when heterogeneity nears $100 \%$. Line of unity, $D^{2}=R^{2}$ is the dotted blue line.

that caution is needed when interpreting meta-analysis before the required DIS has been reached [2-8].

The calculation of HIS and DIS may seem to contrast the $S S$ calculation in a single trial where no adjustment for heterogeneity or diversity is performed. However, Fedorov and Jones [29] advocated the necessity of adjusting SS for heterogeneity arising from different accrual numbers among centres in a multi-centre trial in order to avoid the trial being underpowered. If such an adjustment seems fair for a single trial, it also appears appropriate for a metaanalysis of several trials. As an example, we calculated the DIS to 14,164 participants for a meta-analysis of the effect on mortality of perioperative beta-blockade in patients for non-cardiac surgery (Table 2). This may explain why a recent meta-analysis of seven randomised trials with lowrisk of bias including 11,862 participants indicates, but still does not convincingly show, firm evidence for harm [30]. The actual accrual of 11,862 participants is beyond the HIS of 9,726 participants, but below the DIS of 14,164 participants, and the meta-analysis [30] may still be inconclusive. This suggest that HIS is not a sufficiently adjusted meta-analytic information size. Furthermore, the example demonstrates the important question of the stability of $I^{2}$ and $D^{2}$ beyond a certain number of trials in a meta-analysis as $I^{2}$ was $13.4 \%$ in the meta-analysis after 2,211 participants [19] and has now doubled to $I^{2}=$ $27.0 \%$ after 11,862 accrued participants in the meta-analysis of seven trials with low-risk of bias [30]. The assumption of $I^{2}$ and $D^{2}$ becoming stable after five trials is probably wrong and illustrates the moving target concept, which we have to face doing cumulative meta-analysis as evidence accumulates. Although a moving target may cause conceptual problems, a moving target may be better than no target at all.

The assumption that the IS required for a reliable and conclusive fixed-effect meta-analysis should be as large as the $S S$ of a single well-powered randomised clinical trial to detect or reject an anticipated intervention effect [2-4] may not be necessary in some instances. The statistical information (SINF) required in a meta-analysis could ultimately be expressed as $\operatorname{SINF} \geq \frac{\left(z_{1-\alpha / 2}+z_{1-\beta}\right)^{2}}{\delta^{2}}$ [31], with $\delta$ being the effect size. As SINF is the reciprocal of the variance in the meta-analysis, say $\frac{1}{v_{R}}=\sum_{1}^{k} w_{i}^{*}$, it follows that in meta-analyses with $\sum_{1}^{k} w_{i}^{*} \geq \frac{\left(z_{\alpha / 2}+z_{\beta}\right)^{2}}{\delta^{2}}$, the amount of information may eventually suffice to detect, or reject, an effect size of $\delta$, without yet having reached HIS or DIS. This criterion, however, is not a simple one and may only be fulfilled occasionally. Furthermore, it seems impossible to forecast or even to get an idea of the magnitude of $\sum_{1}^{k} w_{i}^{*}$ in the beginning of a series of trials as well as along the course of trials being performed.

$D^{2}$ offers a number of useful properties compared to $I^{2}$. In contrast to $I^{2}, D^{2}$ reflects the relative variance expansion due to the between trial variance estimate $\hat{\tau}^{2}$ without assuming an estimate of a 'typical' sampling error $\sigma^{2} . D^{2}$ is reduced when the estimate $\hat{\tau}^{2}$ is reduced, even for the same set of trials. In case diversity is larger than inconsistency this may be an indication that total variability among trials in the meta-analysis is even greater than suggested by $I^{2} . I^{2}$ is intrinsically influenced by a potentially overestimated sampling error $\left(\hat{\sigma}_{M}^{2}\right)$, thereby underestimating $\frac{\tau^{2}}{\tau^{2}+\sigma^{2}}$ and inherently placing less weight on large trials with many events. On the other hand a 'typical' sampling error originating from the required information 
size, $\sigma_{D}^{2}$, could be deduced from the $D^{2}$. We would, however, advise great cautiousness in such an attempt. The difference $\left(D^{2}-I^{2}\right)$ reflects the difference of the momentbased and the information size-based 'typical' sampling error estimate. The calculation of diversity and $\left(D^{2}-I^{2}\right)$ may serve as supplementary tools to the assessment of variability in a meta-analysis. $D^{2}$ is a transformation of the variance ratio of the variances from the random-effects model and the fixed-effect model. This variance ratio was a candidate for the quantification of heterogeneity [10].

$D^{2}$ may vary within the same set of trials when different between trial variance estimators $\hat{\tau}^{2}$ are used in the corresponding random-effects model. On the contrary, $I^{2}$ is intimately linked to the specific between trial variance estimator in the DerSimonian-Laird random-effects model as $I^{2}$ by definition is $I^{2}=\frac{Q-(k-1)}{Q}[10]$ and $Q$ is used to estimate a moment-based between trial variance $\hat{\tau}_{D L}^{2}=\frac{Q-(k-1)}{\sum w_{i}-\frac{\sum w_{i}^{2}}{\sum w_{i}}}[15]$. The interpretation of heterogeneity $\frac{\hat{\tau}^{2}}{\hat{\tau}^{2}+\hat{\sigma}^{2}}$ is obviously dependent on the variance estimator $\hat{\tau}^{2}$ as well. An estimate of $\tau^{2}$ is a prerequisite for any random-effects model and the actual estimated value, together with the way $\hat{\tau}^{2}$ is incorporated into the model, actually constitutes the model [32]. Therefore, a quantification of the between-trial variability rather than sampling error which is independent of the specific randomeffects model is impossible, as it is constituted by the between trial variance estimator [32]. $D^{2}$ adapt automatically to different between trial variance estimators [32] while $I^{2}$ is linked to the estimator from the DerSimonianLaird random-effects model.

$D^{2}$ may have some limitations too. The derivation of $D^{2}$ depends on the assumption that the point estimate of the intervention effect in the fixed-effect model and the point estimate of the intervention effect in the random-effects model are approximately equal. Meta-analyses with considerable difference of the point estimate in the fixedeffect model and the point estimate in the random-effects model represent specific problems. Probably more information is needed when $\mu_{F}>\mu_{R}$ since the formula $N_{R}=\frac{v_{R}}{v_{F}} \cdot \frac{\mu_{F}^{2}}{\mu_{R}^{2}} \cdot N_{F}$ yields higher values for $N_{R}$ under the assumption of a constant variance ratio. On the other hand less information may be needed when $\mu_{F}<<\mu_{R}$ since the formula $N_{R}=\frac{v_{R}}{v_{F}} \cdot \frac{\mu_{F}^{2}}{\mu_{R}^{2}} \cdot N_{F}$ then yields lower values for $N_{R}$ under the assumption of a constant variance ratio. However, examples with considerable differences of the point estimates in a fixed- and random-effects model presumably represent meta-analyses of interventions with considerable between trial variance due to small trial bias. The meta-analysis of the effect of magnesium in patients with myocardial infarction is such an example [21] where one large trial totally dominate the result in the fixedeffect model but are unduly down-weighted in the random-effects model. Care should be taken to interpret the random-effects model despite any calculated information size in such a situation. Further, to foresee $a$ priori the size of the difference between $\mu_{F}$ and $\mu_{R}$ seems impossible and the calculation may then degenerate exclusively to a post hoc analysis.

Second, $D^{2}$, though potentially unbiased with respect to information size calculations, could come with a greater variance than $I^{2}$ when both are calculated in the same set of meta-analyses. This latter situation presents a potentially unfavourable 'bias-variance-trade off' but an estimate of its magnitude will have to await simulation studies addressing the issue.

It may seem an advantage that $I^{2}$ is always reported in meta-analysis and therefore readily available to adjust the expected information size. On the other hand $D^{2}=1-\frac{v_{F}}{v_{R}}=1-\left[\frac{\text { width }_{F}}{\text { width }_{R}}\right]^{2}$ is also calculable for metaanalysis of ratio measures (e.g, RR or OR), width $\mathrm{F}_{\mathrm{F}}$ and width $_{R}$ refers to the widths of the confidence intervals for the logarithmic transformed measures in the fixed-effect and the random-effects models, respectively.

Last but not least the decision to pool intervention effect estimates in meta-analysis should be the clinical relevance of any inconsistency or diversity present. The between trial variance, $\tau^{2}$, rather than $I^{2}$ or $D^{2}$, may be the appropriate measure for this purpose [33-35].

The estimation of a required IS for a meta-analysis to detect or reject an anticipated intervention effect on a binary outcome measure should be considered based on reasonable assumptions. Accordingly, it may not be wise to assume absence of heterogeneity in a meta-analysis unless the intervention effect is anticipated to be zero 
$[36,37]$. On the contrary it may be wise to anticipate moderate to substantial heterogeneity (e.g., more than 50\%) in an a priori adjustment of the required IS [37]. The concept of diversity points to the fact that an adjustment based on the experience with inconsistency would result in underestimated heterogeneity and hence an underestimated required IS [37]. Alternatively for a future updated meta-analysis to become conclusive we may apply the actual estimated heterogeneity of the available trials in a meta-analysis as the best we have for the adjustment of the required IS. $D^{2}$ seems more capable than $I^{2}$ in obtaining such an adequate adjustment.

\section{Conclusion}

A quantity to characterise the proportion of between trial variation in any meta-analysis relative to the total model variance of the included trials is needed. Diversity, $D^{2}$, may be such a quantity. $D^{2}$ describes the relative model variance reduction changing from a random-effects model into a fixed-effect model. Diversity may be described as the proportion of the total variance in a random-effects model contributed by the between trial variation despite the chosen between trial variance estimator. Furthermore, $D^{2}$ can adequately adjust the required information size in any random-effects meta-analysis irrespective the metaanalytic model.

\section{List of abbreviations}

$\alpha$ : Risk of type 1 error; $\beta$ : Risk of type 2 error; $A_{R F}$ : Adjustment factor of information size changing from a fixedeffect to a random-effects model; $\forall$ : For any...; $Q$ : Cochran's Q; $D^{2}$ : Diversity; DIS: Diversity adjusted information size $\left(D I S=\frac{S S}{1-D^{2}}\right)$; HIS: Heterogeneity adjusted information size $\left(H I S=\frac{S S}{1-I^{2}}\right)$; $I^{2}$ : Inconsistency factor; $K$ : Number of trials in a meta-analysis; $N_{\mathrm{R}}$ : Required number of participants in a random-effects meta-analysis; $N_{\mathrm{F}}$ : Required number of participants in a fixed-effect meta-analysis; IS: Required number of participants in a meta-analysis; $\mu_{\mathrm{F}}$ : Estimate of the intervention effect in a fixed-effect meta-analysis; $\mu_{\mathrm{R}}$ : Estimate of the intervention effect in a random-effects meta-analysis; OR: Odds ratio; $P C$ : Control event rate; RRR: Relative risk reduction; $S S$ : Sample size in a single randomised clinical trial; $\hat{\sigma}_{D}^{2}$ : Estimate of a typical sampling error considering diversity; $\hat{\sigma}_{M}^{2}$ : Estimate of a typical moment-based sampling error; $\hat{\sigma}_{T}^{2}$ : Mean of estimates of sampling errors in a meta-analysis; $\tau^{2}$ : Estimator of the variance of between trial intervention effect estimates; $\hat{\tau}^{2}$ : Estimate of the variance of between trial intervention effect estimates; $\hat{\tau}_{D L}^{2}$ : DerSimonian-Laird estimate of the variance of between trial intervention effect estimates; $V_{F}$ : The variance in a fixed-effect meta-analysis; $V_{R}$ : The variance in a random-effects metaanalysis; $Z_{1-\alpha / 2}$ : Fractile for $1-\alpha / 2 ; Z_{1-\beta}$ : Fractile for 1- $\beta$.

\section{Conflicts of interests}

The authors declare that they have no competing interests.

\section{Authors' contributions}

JW and KT conceived the idea of an information size adjustment factor and JW devised the concept of diversity, made the mathematical derivations and calculated the examples. KT sat up the simulation program and KT and JW performed the simulations. JW, KT, and CG drafted the first manuscript. KT, JB and CG suggested revisions implemented by JW.

\section{Authors information}

JW is an anaesthesiologist and a trialist working with meta-analysis and trial sequential analysis at the Copenhagen Trial Unit having special interests in perioperative medicine.

KT is a biostatistician working with meta-analysis and trial sequential analysis at the Copenhagen Trial Unit.

$\mathrm{JB}$ is an intern working in paediatrics with meta-analysis and trial sequential analysis.

CG is head of the Copenhagen Trial Unit, Editor-In-Chief of the Cochrane Hepato-Biliary Group, a trialist, and an associate professor at Copenhagen University.

\section{Acknowledgements}

We are grateful to Jørgen Hilden, M.D., associate professor emeritus at the Department of Biostatistics, Copenhagen University, for having critically reviewed a former version of our manuscript. We thank the peer reviewers Rebecca Turner, MSc in statistics and Gerta Rücker, MSc in statistics for helpful suggestions for improvements of the manuscript.

\section{References}

I. Guyatt GH, Mills EJ, Elbourne D: In the era of systematic reviews, does the size of an individual trial still matter? PLoS Medicine 2008, 5(I):e4. doi: 10.1371/jounal.pmed.0050004.

2. Pogue J, Yusuf S: Cumulating evidence from randomized trials: utilizing sequential monitoring boundaries for cumulative meta-analysis. Controlled Clinical Trials 1997, 18:580-93.

3. Pogue J, Yusuf S: Overcoming the limitations of current metaanalysis of randomised controlled trials. Lancet 1998, 35 I (9095):47-52.

4. Devereaux PJ, Beattie WS, Choi PT, Badner NH, Guyatt GH, Villar JC: How strong is the evidence for the use of perioperative beta-blockers in non-cardiac surgery? Systematic review and meta-analysis of randomised controlled trials. BMJ 2005, 33 I (75 I 2):3 |3-2|.

5. Wetterslev J, Thorlund K, Brok J, Gluud C: Trial sequential analysis may establish when firm evidence is reached in a metaanalysis. Journal of Clinical Epidemiology 2008, 6 I (I):64-75. 
6. Brok J, Thorlund K, Gluud C, Wetterslev J: Trial sequential analysis reveals insufficient information size and potentially false positive results in many meta-analyses. Journal of Clinical Epidemiology 2008, 6 I (8):763-9.

7. Thorlund K, Devereaux PJ, Wetterslev, Guyatt G, loannidis JPA, Thabane L, Gluud LL, Als-Nielsen B, Gluud C: Can trial sequential monitoring boundaries reduce spurious inferences from meta-analyses? International Journal of Epidemiology 2008. Doi: I0.1093/iej/dyn I 79.

8. Brok J, Thorlund K, Wetterslev J, Gluud C: Apparently conclusive meta-analyses may be inconclusive-Trial sequential analysis adjustment of random error risk due to repetitive testing of accumulating data in apparently conclusive neonatal metaanalyses. International Journal of Epidemiology 2008. Doi:10.1093/iej/ dyn 188.

9. Altman DG, Deeks JJ: Meta-analysis, Simpson's paradox, and the number needed to treat. BMC Medical Research Methodology 2002, 2:3.

10. Higgins JP, Thompson SG: Quantifying heterogeneity in a metaanalysis. Statistics in Medicine 2002, 21 : 1539-1558.

II. Feinstein AR: Clinical Epidemiology: the Architecture of Clinical Research Philadelphia: W.B. Saunders; 1985: 166.

12. Chow S-C, Shao J, Wang H: Sample Size Calculation in Clinical Research Volume Chapter 8.8.I. Edited by: Shein-Chung Chow. CRC, Taylor \& Francis Group; 2003:204-206.

13. Spiegel MR: Mathematical Handbook of Formulas and Tables I97| [http:/ len.wikipedia.org/wiki/Chebyshev\%27s sum inequality]. Schaum's outline series, McGraw-Hill Book Company

14. Takouche B, Cadarso-Suaréz C, Spiegelman D: Evaluation of old and new tests of heterogeneity in epidemiologic meta-analysis. American Journal of Epidemiology 1999, 150:206-215.

15. DerSimonian R, Laird NM: Meta-analysis in clinical trials. Controlled Clinical Trials 1986, 7:177-I88.

16. Afshari A, Wetterslev J, Brok J, Møller AM: Antithrombin III in critically ill patients. A systematic review with meta-analysis and trial sequential analysis. BMJ 2007, 335(7632): $1219-20$.

17. Al-Inany HG, Abou-Settea AM, Aboulghar M: Gonadotrophinreleasing hormone antagonists for assisted conception. Cochrane Database of Systematic Reviews 2006, 3:CD00 1750.

18. Soll RF: Prophylactic natural surfactant extract for preventing morbidity and mortality in preterm infants. The Cochrane Database of Systematic Reviews 1997, 4:CD0005 I I.

19. Wetterslev J, Juul $A B$ : Benefits and harms of perioperative beta-blockade. Best Practice and Research of Clinical Anesthesiology 2006, 20:285-302

20. Bury RG, Tudehope D: Enteral antibiotics for preventing necrotizing enterocolitis in low birthweight or preterm infants. The Cochrane Database of Systematic Reviews 2000, 2:CD000405. DOI: 10.1002/1465/858.CD000405.

21. Li J, Zhang M, Egger M: Intravenous magnesium for acute myocardial infarction. Cochrane Database of Systematic Reviews 2007. 2: CD002755.

22. Meyhoff CS, Wetterslev J, Jorgensen LN, Henneberg SW, Simonsen I, Pulawska T, Walker LR, Skovgaard N, Heltø K, Gocht-Jensen P, Carlsson PS, Rask H, Karim S, Carlsen CG, Jensen FS, Rasmussen LS, the PROXI Trial Group: Perioperative oxygen fraction - effect on surgical site infection and pulmonary complications after abdominal surgery: a randomized clinical trial. Rationale and design of the PROXI-Trial. Trials 2008, 9(I):58.

23. Gluud LL: Bias in clinical intervention research. American Journal of Epidemiology 2006, 163:493-50I.

24. Chan AW, Hrobjartsson A, Haahr MT, Gøtzsche PC, Altman DG Empirical evidence for selective reporting of outcomes in randomized trials: comparison of protocols to published articles. Journal of American Medical Association 2004 29 I(20):2457-2465

25. Chan AW, Altman DG: Identifying outcome reporting bias in randomised trials on PubMed: review of publications and survey of authors. BM] 2005, 330(7494):753

26. Wood L, Egger M, Gluud LL, Schulz KF, Jüni P, Altman DG, Gluud C, Martin RM, Wood AJ, Sterne JA: Empirical evidence of bias in treatment effect estimates in controlled trials with different interventions and outcomes: meta-epidemiological study. BMJ 2008, 336(7644):60I-5.

27. Montori VM, Devereaux PJ, Adhikari NK, Burns KE, Eggert CH, Briel $M$, Guyatt G: Randomized trials stopped early for benefit: a systematic review. Journal of American Medical Association 2005, 294(I7):2203-2209.

28. Flather MD, Farkouh ME, Pogue JM, Yusuf S: Strengths and limitations of meta-analysis: larger studies may be more reliable. Controlled Clinical Trials 1997, 18(6):568-579.

29. Fedorov V, Jones B: The design of multicentre trials. Statistical Methods in Medical Research 2005, 14:205-248.

30. Bangalore S, Wetterslev J, Pranesh S, Sawhney S, Gluud C, Messerli $\mathrm{FH}$ : Perioperative beta blockers in patients having non-cardiac surgery: a meta-analysis. Lancet 2008, 372(9654):1962-76.

31. Jennison C, Turnbull BW: Group sequential methods with application to clinical trials Volume Chapter III. Chapman \& Hall/CRC; 2000:49.

32. Sidik K, Jonkman JN: A comparison of heterogeneity variance estimators in combining results of studies. Statistics in Medicine 2007, 30;26(9): |964-8|.

33. Rücker G, Schwarzer, Carpenter JR, Schumacher M: Undue reliance on $I^{2}$ in assessing heterogeneity may mislead. BMC Medical Research Methodology 2008, 8:79. doi:I0.1 I 86/I47I-2288-8-79.

34. Higgins JP: Commentary: Heterogeneity in meta-analysis should be expected and appropriately quantified. International Journal of Epidemiology 2008, 37: I I58-1 I60.

35. Rücker G, Schwarzer G, Carpenter JR, Schumacher M: Are large trials less reliable than small trials? Letter to the editor. Journal of Clinical Epidemiology 2009, 62:886-889.

36. Ioannidis JP, Trikalinos TA, Zintzaras E: Extreme between-study homoge-neity in meta-analyses could offer useful insights. Journal of Clinical Epidemiology 2006, 59( 10): 1023-32.

37. loannidis JP, Patsopoulos NA, Evangelou E: Uncertainty in heterogeneity estimates in meta-analyses. BMJ 2007 335(7626):914-6.

\section{Pre-publication history}

The pre-publication history for this paper can be accessed here:

http://www.biomedcentral.com/1471-2288/9/86/prepub
Publish with BiolMed Central and every scientist can read your work free of charge

"BioMed Central will be the most significant development for disseminating the results of biomedical research in our lifetime. "

Sir Paul Nurse, Cancer Research UK

Your research papers will be:

- available free of charge to the entire biomedical community

- peer reviewed and published immediately upon acceptance

- cited in PubMed and archived on PubMed Central

- yours - you keep the copyright

Submit your manuscript here:

http://www.biomedcentral.com/info/publishing_adv.asp
BioMedcentral 